\title{
Therapeutic apheresis in peripheral and retinal circulatory disorders
}

\author{
Alfonso Ramunni • Paola Brescia • Giuseppina De Fino • \\ Giovanni Piscopo $\cdot$ Loreto Gesualdo
}

Published online: 25 February 2012

(C) The Author(s) 2012. This article is published with open access at Springerlink.com

\begin{abstract}
In microcirculation disorders, the therapeutic apheresis seems to have two different effects. The first, achieved after only a few sessions, is acute, consisting of drastic reduction of blood viscosity and obtained with the use of low-density lipoprotein (LDL) apheresis, rheopheresis, or fibrinogen apheresis. The second effect is long term, or chronic, and needs to be evaluated after a long course of treatment. The mechanisms underlying the chronic effect are still objects of debate and take into account the pleiotropic effects of apheresis. However, it is likely that the acute effect of apheresis mainly influences the functional components of the vascular damage, and so the derived rheological benefit might last only for a short period. The chronic effect, on the contrary, by acting on the morphological alterations of the vascular walls, requires the apheresis treatment to be prolonged for a longer period or even cycles of treatment to be programmed.
\end{abstract}

Keywords LDL apheresis · Rheopheresis ·

Microcirculation disorders $\cdot$ Peripheral arterial disease

Dr. A. Ramunni $(\bowtie)$

Policlinico Universitario Consorziale, 70124 Bari, Italy

e-mail: a.ramunni@nephro.uniba.it

Dr. A. Ramunni · G. De Fino · G. Piscopo · L. Gesualdo Section of Nephrology, Department Emergency and Organ

Transplantation, University of Bari, Bari, Italy

P. Brescia

"C Poma" Hospital, Mantova, Italy

\section{Introduction}

In the last years, different strategies of therapeutic apheresis have been applied to treat various microcirculation disturbances. In the vast majority of cases reported in literature, the number of apheresis sessions considered necessary to achieve a positive outcome is extremely small. In sudden hearing loss (SHL), a disease affecting the inner ear vascularization, just one session was able to improve the hearing function [1].

It has been presumed that the reasons justifying such a rapid effect are linked to the rheological properties of therapeutic apheresis and its capacity to reduce the concentrations of some plasma proteins closely connected with hemorheology [2].

However, it is now becoming evident that long-term treatment with therapeutic apheresis, especially chronic therapy with low-density lipoprotein (LDL) apheresis in patients suffering from familial hypercholesterolemia, is able to reduce atherosclerotic plaque and to increase the vessel lumen, thus acting to improve morphological alterations of the vessels and showing an effect that extends far beyond the purely hemorheological one.

In Mellwig's experience [3], for instance, the myocardial perfusion under LDL apheresis, evaluated by PET, was considerably improved after the first session of LDL apheresis, but showed a more marked increase after 9 months of treatment.

Therefore, it seems likely that therapeutic apheresis is capable of achieving two different effects: a rapid effect, also called acute effect that takes place very rapidly, even a few hours after the end of the first session, and a slow one, also called chronic effect, which takes several weeks or months to develop. 
The acute effect may be attributed to the reduction in whole blood viscosity whereas the chronic effect is more difficult to explain.

\section{Acute effect}

The first experiences of an acute effect of therapeutic apheresis were reported in an application in SHL. In a frequently quoted article by Suckfull [1], an improved hemorheological status induced by a single session of LDL apheresis was capable of significantly improving speech perception, especially in patients with higher levels in fibrinogen (more than $8.68 \mathrm{mmol} / \mathrm{L})$.

The greater importance attributed to the reduction of fibrinogen as a means of predicting patients' outcome in SHL paved the way for the use of rheopheresis in the treatment of this disease. In their study, Mösges and colleagues [4] proved that rheopheresis could be an effective treatment option for SHL, demonstrating that two sessions of this apheresis strategy within 3 days lasting for about $2 \mathrm{~h}$ each could be used to replace a 10-day infusion regimen. Ullrich and colleagues [5] confirmed the role of fibrinogen in improving hemorheology by means of fibrinogen apheresis, which they used as a therapeutic procedure for the treatment of SHL. In this study also, the authors administered only few sessions of apheresis, three at the most. The main auditory improvement was evident directly after the end of treatment and the reduction in relative mean hearing loss from the original $35.2 \mathrm{~dB}$ to $11.8 \mathrm{~dB}$ was highly significant.

There is another similar disease affecting a sensory organ in which therapeutic apheresis has been shown to be efficacious, namely nonarteritic anterior ischemic optic neuropathy (NAION). The main pathogenetic mechanism of NAION seems to be the result of an insufficient blood supply to the optic nerve head, due to the involvement of the posterior ciliary arteries. Despite traditional treatment, the disease has a substantially stable course over time and may even get worse. Therefore, NAION has been an excellent field of application to verify the efficacy of therapeutic apheresis, much better than SHL where a spontaneous recovery is frequently observed. In two pilot studies, the use of three sessions of LDL apheresis determined a clear improvement in the functional data for the affected eyes [6, 7]. In fact, the scotomatous portion of the visual field regressed already after the first session of LDL apheresis and further regressed after the third. To understand the mechanisms underlying such a rapid effect, in another study we correlated the mean deviation value change that is a more reliable index of an improvement of the visual field, together with a reduction of some markers of endothelial dysfunction (including fibrinogen and lipids). We found a highly significant correlation, although no parameter was shown to have a prevalent influence with respect to the others [8].

Taking into account another microcirculation disorder, peripheral arterial disease (PAD), a single session of fibrinogen apheresis was shown to be able to improve the clinical picture with a significant increase in both total and pain-free walking distance, as a result of drastic reduction in serum fibrinogen concentration [9].

In all these experiences, the different apheresis strategies have in common a rapid drop in macromolecule concentrations that has an effect on the blood rheology. We know that hemorheology is determined by the viscosity of the plasma and the whole blood and by the erythrocyte deformability and tendency to aggregate. Otto and colleagues [10] demonstrated that the effect of fibrinogen on plasma viscosity seems to be more pronounced than the influence of lipoprotein concentration. In fact, because of its molecular weight and more importantly its size, the fibrinogen has an important role in determining the plasma viscosity, even if it accounts for only $4 \%$ of the plasma protein content. Moreover, fibrinogen has often been described as the most important protein in physiological and pathological red blood cells (RBC) aggregation, thus increasing the whole blood viscosity. The lipoproteins also play a major role in RBC aggregation [11]. RBC deformability, as an additional actor, has a fundamental importance in determining whole blood viscosity. The shape of RBC could be changed by the addition of lipoproteins to a suspension of RBCs [12], and the loss of RBCs deformability does not allow the cells to pass through a capillary vessel, thereby stopping the blood supply.

To summarize, the mediators of blood viscosity that can influence RBC deformability, RBC aggregation, and plasma viscosity are essentially the fibrinogen and lipoproteins. By lowering the concentrations of these macromolecules, the LDL apheresis, rheopheresis, or fibrinogen apheresis reduce the blood viscosity and therefore the resistance to flow, according to the Hagen-Poiseuille law. For these reasons, these three procedures could have an effect-I would say a rapid effect - on the clinical picture of microcirculation disorders, such as SHL, NAION, and PAD. However, we do not know how long these effects on hemorheology last. In other words, is acting on hemorheology, with so few sessions of apheresis, or even with a single session, enough to bring about a complete recovery in our patients?

In NAION, we aimed to answer this question by assessing the conditions of a group of patients 6 months after they had been treated with LDL apheresis [13]. The mean deviation of the visual field resulted statistically improved at discharge as compared with admission. However, the improvement did not persist up to 6 months, and there was a return to the baseline values recorded at hospital admission. In a study of uremic patients affected by PAD and treated 
with LDL apheresis according to a regimen involving 5 sessions in 5 weeks, the blood flow in the peripheral microcirculation of the lower limbs, examined by laser Doppler, which improved after the first, third, and fifth session, had returned to baseline values before the start of the third and fifth sessions [14].

It seems likely, therefore, that a rapid but short effect is to be expected after few sessions of therapeutic apheresis, since the rheological advantage of apheresis is related to the rebound kinetics of the rheologically relevant plasma proteins. For these reasons, the effectiveness of therapeutic apheresis seems to last only as long as it is applied, with benefits that persists for only a few months after it is stopped.

\section{Chronic effect}

Nevertheless, long-term treatment with therapeutic apheresis has been shown to induce a suppression of the progression of atherosclerotic lesions and eventually also a regression of atherosclerotic lesions, thus acting to improve morphological alterations of the vessels. After 1 year followup, the coronary angiograms of patients receiving chronic treatment with LDL apheresis displayed an increase in the minimal lumen diameter and a reduction of the area of plaque [15]. A long-term treatment with LDL apheresis was able to induce a reduction of coronary calcifications [16] in a patient affected by familial hypercholesterolemia. After 10 sessions of LDL apheresis, a complete occlusion of the anterior tibial artery improved to a partial stenosis, with a nearly complete healing of a decubitus [17].

Thus, in the long-term treatment, the LDL apheresis seems to influence actions going far beyond the hemorheological ones, that are confined to the first phases of the treatment only [18]. It is known that apart from the direct lipid reduction effect, the LDL apheresis has been shown to have an extensive series of other actions, the so-called pleiotropic effects of LDL apheresis [19]. Among these, it is possible to consider a possible influence of LDL apheresis on the number of circulating endothelial progenitor cells, which can act directly on the vascular walls and repair vascular lesions [20].

The differences between the acute and chronic effects of therapeutic apheresis are therefore a field which is still open for discussion, where it is essential to decide the timing and duration of treatment.

In one of our experiences, for instance, we decided to treat the two patients with PAD, who were in imminent danger of amputation, by adopting the same schedule of treatment and then stopping the apheresis in one patient and continuing the procedure in the other one, in order to establish the best timetable for this therapeutic approach. The patients underwent 22 sessions of rheopheresis over 4 months; then the first patient continued the apheresis at the rate of one session a week for another year while the second patient remained untreated. Ischemic lesions, as evaluated by photographs, showed a nearly complete healing at the end of the cycle of apheresis in both patients. The improvement persisted in the first patient during the year of apheresis, while a new ulcer reappeared just 2 months after the end of apheresis. On the contrary, in the second patient the situation was still substantially unchanged 14 months after the end of apheresis.

These observations provide an example of the effectiveness of rheopheresis even in the presence of ulcers treated with a regimen of 22 sessions in 4 months and underline the necessity to personalize the treatment in order to avoid the reappearance of the previous clinical state shortly after the treatment is stopped.

\section{Conclusion}

In conclusion, what we still need to explore further is the timing and duration of the use of therapeutic apheresis. It is reasonable to believe that few sessions of apheresis may act principally on the functional components of the vascular damage and the derived rheological benefit might last only for a short period (for instance, a reduction of pain or an increase in the walking distance for PAD). On the contrary, if we aim to operate on the morphological components of the vascular damage (for example, to heal an ulcer and to prevent its reappearance after a short time in our patients), it seems likely that we need to prolong the apheresis treatment for a longer period or even to program several cycles of treatment.

Acknowledgments This article is part of a supplement sponsored by an unrestricted educational grant from B. Braun and Fresenius Medical Care.

Conflict of interest The authors declare that they have no conflict of interest.

Open Access This article is distributed under the terms of the Creative Commons Attribution License which permits any use, distribution, and reproduction in any medium, provided the original author(s) and the source are credited.

\section{References}

1. Suckfüll M (2002) Hearing loss study group fibrinogen and LDL apheresis in treatment of sudden hearing loss: a randomized multicenter trial. Lancet 360(9348):1811-1817

2. Schuff-Werner P, Holdt B (2002) Selective hemapheresis, an effective new approach in the therapeutic management of disorders associated with rheological impairment: mode of action and possible clinical indications. Int J Artif Organs 26(2):117-123 
3. Mellwig KP, Baller D, Schmidt HK, V Buuren F, Wielepp JP, Burchert W, Horstkotte D (2003) Myocardial perfusion under H.E.L.P.--apheresis. Objectification by PET. Z Kardiol 92(Suppl 3):III30-III37

4. Mösges R, Köberlein J, Heibges A, Erdtracht B, Klingel R, Lehmacher W (2009) RHEO-ISHL study group. Rheopheresis for idiopathic sudden hearing loss: results from a large prospective, multicenter, randomized, controlled clinical trial. Eur Arch Otorhinolaryngol 266(7):943-953

5. Ullrich H, Kleinjung T, Steffens T, Jacob P, Schmitz G, Strutz J (2004) Improved treatment of sudden hearing loss by specific fibrinogen aphaeresis. J Clin Apher 19(2):71-78

6. Ramunni A, Giancipoli G, Saracino A, Guerriero S, Saliani MT, Gentile MC, Sborgia C, Coratelli P (2004) LDL-apheresis in acute anterior ischemic optic neuropathy. Int $\mathrm{J}$ Artif Organs 27(4):337-341

7. Ramunni A, Giancipoli G, Guerriero S, Lapenna L, Saracino A, Saliani MT, Capurso A, Sborgia C, Coratelli P (2005) LDLapheresis accelerates the recovery of nonarteritic acute anterior ischemic optic neuropathy. Ther Apher Dial 9(1):53-58

8. Ramunni A, Ranieri G, Giancipoli G, Guerriero S, Ria R, Saliani MT, Sborgia L, Ranieri P, Coratelli P (2006) Is the efficacy of LDL apheresis in ischemic optic neuropathy linked to a reduction in endothelial activation markers? Blood Purif 24(4):405-412

9. Ramunni A, Brescia P, Quaranta D, Plantamura M, Ria R, Coratelli $\mathrm{P}$ (2007) Fibrinogen apheresis in the treatment of peripheral arterial disease. Blood Purif 25(5-6):404-410

10. Otto C, Richter WO, Schwandt P (2000) Contribution of fibrinogen and lipoproteins to plasma viscosity in hypercholesterolemia and hypertriglyceridemia: evaluation by selective depletion of low-density lipoproteins or fibrinogen. Metabolism 49(6):810-813

11. Moriarty PM, Gibson CA (2005) Association between hematological parameters and high-density lipoprotein cholesterol. Curr Opin Cardiol 20(4):318-323

12. Doi H, Kugiyama K, Ohgushi M, Sugiyama S, Matsumura T, Ohta Y, Oka H, Ogata N, Hirata A, Yamamoto Y, Yasue H (1999) Membrane active lipids in remnant lipoproteins cause impairment of endothelium-dependent vasorelaxation. Arterioscler Thromb Vasc Biol 19(8):1918-1924
13. Guerriero S, Giancipoli G, Cantatore A, Sacco G, Brescia P, Saliani MT, Ramunni A (2009) LDL apheresis in the treatment of non-arteritic ischemic optic neuropathy: a 6-month followup study. Eye (Lond) 23(6):1343-1344

14. Ebihara I, Sato T, Hirayama K, Seki M, Enami T, Kawahara H, Niwayama J, Miyahara T, Shibata M, Maeda N, Kurosawa T, Yamagata K, Sanaka T (2007) Blood flow analysis of the head and lower limbs by the laser Doppler blood flowmeter during LDL apheresis. Ther Apher Dial 11(5):325-330

15. Matsuzaki M, Hiramori K, Imaizumi T, Kitabatake A, Hishida H, Nomura M, Fujii T, Sakuma I, Fukami K, Honda T, Ogawa H, Yamagishi M (2002) Intravascular ultrasound evaluation of coronary plaque regression by low density lipoprotein apheresis in familial hypercholesterolemia: the Low Density Lipoprotein-Apheresis Coronary Morphology and Reserve Trial (LACMART). J Am Coll Cardiol 40(2):220-227

16. Ramunni A, Morrone LF, Baldassarre G, Montagna E, Saracino A, Coratelli P (2003) Effectiveness of long-term heparin-induced extracorporeal LDL precipitation (HELP) in improving coronary calcifications. Int J Artif Organs 26(3):252-255

17. Kamimura M, Matsuo M, Miyahara T, Kimura K, Matsumoto K, Nakaya T, Abe T, Akizawa T (2002) Improvements in artery occlusion by low-density lipoprotein apheresis in a patient with peripheral arterial disease. Ther Apher 6(6):467-470

18. Pusl T, Broedl UC, Parhofer KG, Otto C (2009) Long-term LDL apheresis does not stably improve hemorheology in hypercholesterolemic patients with coronary artery disease. Clin Hemorheol Microcirc 41(2):137-142

19. Ramunni A, Burzo M, Vernò L, Brescia P (2009) Pleiotropic effects of LDL apheresis. Atheroscler Suppl 10(5):53-55

20. Ramunni A, Brescia P, Dambra P, Capuzzimati L, Ria R, De Tullio G, Resta F, Russi G, Vacca A, Coratelli P (2010) Effect of low-density lipoprotein apheresis on circulating endothelial progenitor cells in familial hypercholesterolemia. Blood Purif 29(4):383-389 